\title{
Generalization decrements: Further support for flexibility in stimulus processing
}

\author{
AnNa Thorwart and Harald Lachnit \\ Philipps-Universität Marburg, Marburg, Germany
}

\begin{abstract}
Thorwart and Lachnit (2009) found reliable symmetrical decrements in two generalization tasks: Removing an already trained component from a compound did not result in larger decrements than adding a new one did. In two contingency learning experiments, we investigated first whether time pressure during stimulus processing, as well as the degree of perceptual grouping, was effective in controlling the symmetry of the decrements (Experiment 1); and second, whether the symmetry was affected by the causal versus predictive nature of the relationship between the cue and the outcome (Experiment 2). The experiments generated unexpected results, since both revealed asymmetrical decrements independent of the manipulations introduced. They therefore demonstrate that more research is needed in order to understand the variables influencing stimulus representation in human associative learning.
\end{abstract}

Traditionally, many well-known associative learning phenomena have been explained in a so-called elemental manner, in which it is assumed that stimulus components are represented as separate entities, and that the overall associative strength of a compound - for instance, $\mathrm{AB}$ - is based on the algebraic sum of the associative strengths of components A and B (e.g., Mackintosh, 1975; Pearce \& Hall, 1980; Rescorla \& Wagner, 1972). On the other hand, there is considerable evidence that humans as well as animals can successfully handle discrimination problems that cannot be solved in such a purely elemental manner. Therefore, more recent elemental models like the replaced elements model (REM; Wagner, 2008) and the model of Harris (2006) assume, first, that a component presented within a compound may not activate exactly the same entities and associations as when presented on its own and, second, that the degree of overlap between these two instances of the component's representation is somewhat variable.

Others have suggested instead that stimuli are always processed configurally. In this view, a compound $A B$ maps onto a representational entity, a so-called configural unit, which is distinct from its components' representation. Only this configural unit enters into an association with the outcome. Furthermore, with respect to behavior, it is assumed that generalization between such entities takes place on the basis of their similarity (e.g., Pearce, 1994).

These rival theories can be tested empirically in a range of different ways. First, for certain discrimination problems, these theories clearly differ in their predictions about which discriminations should be acquired more rapidly (see, e.g., Harris, Livesey, Gharaei, \& Westbrook, 2008;
Kinder \& Lachnit, 2003; Lachnit, Schultheis, König, Üngör, \& Melchers, 2008; Redhead \& Pearce, 1995; Thorwart, Glautier, \& Lachnit, 2010). Second, these theories differ with respect to responding to new stimuli in a test after acquisition (e.g., summation-Glautier, Redhead, Thorwart, \& Lachnit, 2010; Lachnit, 1988; Myers, Vogel, Shin, \& Wagner, 2001; Pearce, Aydin, \& Redhead, 1997; generalization decrement-Brandon, Vogel, \& Wagner, 2000; Glautier, 2004; González, Quinn, \& Fanselow, 2003; Thorwart \& Lachnit, 2009; Wheeler, Amundson, \& Miller, 2006). Most current theories of associative learning assume that stimuli are invariably processed in the same way. As a consequence, the question whether stimuli are processed elementally or configurally is usually discussed in an "either/or" manner (for recent reviews, see Pearce \& Bouton, 2001; Wasserman \& Miller, 1997). However, the empirical results observed so far have been mixed (even within one study; e.g., Giurfa, Schubert, Reisenman, Gerber, \& Lachnit, 2003). One explanation could be that there is flexibility in the way in which humans and animals deal with discrimination problems (for details, see Melchers, Shanks, \& Lachnit, 2008).

In light of this potential flexibility, we (Thorwart \& Lachnit, 2009) investigated generalization decrementwhere support for elemental theories had hitherto been found - in order to determine factors that might influence the kind of processing there, too. We used two different generalization tasks introduced by Brandon et al. (2000). In the added-cue task, a new stimulus component is added to the previously trained stimulus $(\mathrm{AB}+\rightarrow \mathrm{ABX})$, whereas in the removed-cue task, a stimulus component is removed from the previously trained stimulus $(\mathrm{EFG}+\rightarrow \mathrm{EF})$. The predictions of the rival models were simulated using sev-

A. Thorwart, anna.thorwart@sydney.edu.au 
eral MATLAB-based programs (Schultheis, Thorwart, \& Lachnit, 2008a, 2008b; Thorwart, Schultheis, König, \& Lachnit, 2009). Pearce's (1994) configural model predicts symmetrical decrements (i.e., the amount of decrement produced by testing $\mathrm{ABX}$ after training with $\mathrm{AB}$ will be the same as that produced in testing with $\mathrm{EF}$ after training with EFG). On the other hand, both elemental theories (Harris, 2006; Wagner, 2008) predict asymmetrical generalization decrements: Removal of components always results in a larger decrement than adding does. This prediction of asymmetry remains unaffected by the supposed variability in the components' representations.

Previously, experiments intended to compare these decrements reported only asymmetrical decrements in human (Glautier, 2004; Wheeler et al., 2006), as well as in nonhuman learning (Brandon et al., 2000; González et al., 2003) $)^{1}$. Guided by the question "Under which conditions is processing best described by configural theories, and under which conditions is it best described by elemental theories?", we attempted to investigate which manipulation of the stimuli is effective in producing symmetrical generalization decrements, and thus, configural processing. In the first experiment of Thorwart and Lachnit (2009), the Gestalt principle of common fate was used, according to which objects moving with the same speed in the same direction become perceptually grouped and are seen as belonging together (Palmer, 1999). Five or two dots differing in color were presented moving across a black screen for $2 \mathrm{sec}$, either all in the same direction or in different directions. However, symmetrical decrements were observed in both conditions. In Experiment 2 of Thorwart and Lachnit, we "froze" the colored dots on their starting position (i.e., the dots were presented at the starting positions without movement). Again, however, symmetrical generalization decrements were observed. Experiment 3 included a between-subjects factor of position: In Group Variable, the dots were randomly positioned, as in Experiment 2; in Group Fixed, the dots were presented on three selected positions in the middle of the screen. Again, symmetrical decrements were observed under all conditions.

We discussed three potential reasons for this reliable discrepancy between our results and those of others, two of which (duration of stimulus presentation, cover story of the task) we will focus on in the experiments of the present article. First, from other studies in our lab comparing causal learning and classical conditioning, we have clues that the stimulus duration and, thus, time pressure may influence stimulus processing (Lachnit et al., 2010). In all three experiments of Thorwart and Lachnit (2009), stimulus presentation had been limited to $2 \mathrm{sec}$, whereas the stimulus duration in Wheeler et al.'s (2006) causal learning experiments, for instance, had been self-paced by the participants. Hence, reducing the time pressure by a prolonged stimulus presentation may result in asymmetrical decrements. Second, even though all mentioned human studies were contingency learning experiments, they used different cover stories. The food-poisoning scenario of Wheeler et al., as well as the air pollution scenario of Glautier (2004), are causal scenarios. The participants had to rate the likelihood that the meals caused food poisoning, or how much pollution was caused by an airplane, respectively. In the power plant scenario of Thorwart and Lachnit, no statement was made about the causal relationship between the dots and the cooling water's temperature. Pineño, Denniston, Beckers, Matute, and Miller (2005) observed reliable overshadowing only between causes, and only when the test question was causal. Because the removed-cue task measures only overshadowing, the symmetry in our experiments may have been due to reduced overshadowing (i.e., relatively small decrements) in the removed-cue task, because of the predictive scenario.

\section{EXPERIMENT 1}

The present experiment explicitly manipulated stimulus duration between participants and used a causal scenario that might possibly enhance the possibility of asymmetrical decrements. As in Experiment 1 of Thorwart and Lachnit (2009), the influence of perceptual grouping by motion on the stimulus processing was investigated. Again, the cues were small, differently colored dots moving across a black screen. The color of the dots was critical in solving the required discrimination, whereas motion, number of dots, and duration were irrelevant for predicting the outcome. Thus, participants received four stimulus compounds during training: two compounds with two dots and two with three dots, one of each followed by the outcome $(\mathrm{AB}+, \mathrm{CD}-, \mathrm{EFG}+, \mathrm{HIJ}-$, each letter referring to a dot of a specific color). In the test stage, a new dot was added to the two-dot compounds (ABX, CDY), whereas one dot was removed from the three-dot compounds (EF/EG/FG, HI/HJ/IJ). To evaluate the models' predictions, generalization decrements for the reinforced stimuli in the removed-cue (EFG - EF/EG/FG) and added-cue $(\mathrm{AB}-\mathrm{ABX})$ tasks were compared within subjects. $\mathrm{A}$ between-subjects factor controlled whether the dots were presented as long as in Thorwart and Lachnit $(2 \mathrm{sec})$ or four times as long $(8 \mathrm{sec})$. The kind of motion varied as a second between-subjects factor: In the separated-motion conditions, the direction of motion was randomly chosen for each dot and trial (with some restrictions); in the grouped-motion conditions, all dots were moving in the same direction (chosen randomly for each trial). In Experiment 1 of Thorwart and Lachnit, we had hypothesized that perceptually grouped stimuli should be processed according to configural principles, whereas nongrouped stimuli were prone to elemental processing. However, we had no chance to observe any influence of perceptual grouping on processing since there already had been symmetrical decrements - that is, configural processing - in the separated-motion condition. The resultant floor effect should be avoided in the present experiment, at least in the condition with the prolonged stimulus duration where we expect asymmetrical decrements (elemental processing) for the separate-motion group. Additionally, the instruction introduced for all of the groups a medical cover story with a causal link between the dots (bacteria in the 
blood) and the outcome (disease). This causal cover story should increase overshadowing in the removed-cue task and, again, the likelihood of asymmetrical decrements in the separated-motion condition. Therefore, the risk of a floor effect is further reduced.

\section{Method}

Participants. We recruited 159 participants from the university campus through posted advertisements. They received course credits or were paid. Participants were included in further analysis if they solved the discrimination at the end of the training. To be included, the rating in the last two trials of training had to be equal or higher than 5 for the reinforced stimuli and equal or lower than 4 for the nonreinforced stimuli (these numbers refer to scores on an 8-point scale; see below). The remaining 126 participants (30 men and 96 women) were from 19 to 40 years of age, with a mean age of 22.53 . In four groups - separated-short, separated-long, grouped-short, and grouped-long-33, 33, 31, and 29 participants were tested, respectively.

Apparatus and Stimuli. The experiment took place in labs of the Department of Psychology. In the labs, stimuli were presented on 17-in. color monitors running in a 1,280 (width) $\times 1,024$ (height) pixel resolution and with a $75-\mathrm{Hz}$ frame rate.

Different colored dots (17 pixels) were used as the cues. Twelve different colors were needed for the whole experiment (RGB: 64 204 255; 53169 55; 23450 24; 14736 207; 23829 90; 187212 65; 23195 217; 6949 181; 255157 0; 253228 111; 00 224; 200255 200). The colors were assigned randomly for each participant, but the separated-motion and grouped-motion groups were matched. The dots moved across a central $750 \times 750$ pixel area with a speed of $1 \mathrm{pixel} /$ frame. One trial consisted of 150 frames in the short- and 600 frames in the long-stimulus-duration condition, resulting in 2 and $8 \mathrm{sec}$, respectively. (For 13 participants of the separated-long group, the frame rate was $60 \mathrm{~Hz}$ due to a procedural error, resulting in additionally prolonged stimulus duration of $10 \mathrm{sec}$. ANOVAs of the generalization decrement of this group including this factor revealed no significant influence: $F<1.2$.) The starting points and trajectories of the dots were chosen randomly for each participant and each trial. The only restriction was that the distance between the dots had to be greater than 25 pixels at any time during movement. In the separated-motion conditions, one trajectory for each dot was chosen randomly per trial, whereas in the grouped-motion conditions, one trajectory common for all dots per trial was computed. The background was black during the whole experiment. Stimulus generation and experimental control were accomplished by MATLAB 7, using the Psychophysics Toolbox 2.54 (Brainard, 1997).

Procedure. At the start of the experiment, each participant read the following instructions (in German) on the screen:

Dear participant! Thank you for your readiness to participate in this experiment.

In the following experiment, you will learn which bacteria in the blood are causal for a new illness. Please imagine that you are working in a medical laboratory. Recently there were several cases of this new illness. In order to find the cause for this illness, you use a new procedure for investigating bacteria in blood samples.

At any one time, you will see the sample of one test person on the screen. Afterward please indicate whether the bacteria in this blood sample cause a disease, and therefore whether the test person is sick or not. Perhaps you will not be perfectly sure with your decision; therefore, an 8-point answer scale is available, ranging from " 1 = definitely not ill" to " $8=$ definitely ill." After you have delivered your rating, you will receive feedback whether the disease occurred or not. At the beginning you will have to guess but after a while you will learn what causes a disease. It doesn't matter how fast you answer; therefore, take the time you need. If you have any question, please ask now. Otherwise, please start the experiment by clicking on the "next" button.

Each trial started with a pattern of dots moving across the black screen. After the dots disappeared after either 2 or $8 \mathrm{sec}$, the question "Are the bacteria in this blood sample causing a disease?" was shown, together with an 8-point rating scale with the anchors marked "definitely not ill" and "definitely ill." After the participants marked one of the check boxes, they received feedback indicating whether or not the patient was ill. Before the test stage, a further instruction told the participants that during the next experimental phase there would be additional new stimuli and no more feedback.

The training stage comprised 15 trials of $\mathrm{AB}+, \mathrm{CD}-, \mathrm{EFG}+$, and HIJ-, respectively, with each letter corresponding to one color; "+" indicates that the patient was ill, "-" that he was not. The test stage consisted of 36 test trials: three trials each of $\mathrm{AB}, \mathrm{CD}, \mathrm{EFG}$, HIJ, ABX, and CDY, respectively, and one trial each of EF, GH, EG, HI, IJ, HJ, A, B, C, D, E, F, G, H, I, J, X, and Y. Thus, every dot of the trained stimulus EFG was removed once. Test trials consisting only of one dot were included for the sake of completeness. However, since the grouped-motion versus separated-motion manipulation cannot be implemented with only one dot, those trials cannot be properly compared with the other trials. Trial sequences were randomized, and the same sequences were then used in all groups.

\section{Results and Discussion}

For all statistical analyses, $\alpha$ was set to .05 , and HuynhFeldt's (1976) correction of the degree of freedom was used where appropriate.

Figure 1 displays the mean ratings for the relevant stimuli in the test stage. The upper panels show the results of both groups with prolonged stimulus duration of $8 \mathrm{sec}$, the lower panels for the groups with the previously used, shorter stimulus duration of $2 \mathrm{sec}$. The groups with dots moving in different directions (separated motion) are shown on the left side, and groups with dots moving in the same direction (grouped motion) on the right side. Within each panel, the black bars represent the mean rating of the previously trained stimulus (AB for the added-cue task on the left side, EFG for the removed-cue task on the right side), whereas the white bars represent the mean rating of the corresponding test stimulus ( $\mathrm{ABX}$ and $\mathrm{EF} / \mathrm{EG} / \mathrm{FG}$, respectively). First of all, the results did not differ between the groups. In all groups, the ratings of test stimuli were lower than were those of the reinforced training stimuli. Moreover, the decrement seemed to be larger for the removed-cue than for the added-cue task, independent of the manipulation of the stimulus duration or the motion. This was confirmed by a $2 \times 2 \times 3 \times 2 \times 2$ ANOVA including the within-subjects factors cue (trained vs. test), task (added vs. removed), and block (1-3), and the between-subjects factors stimulus duration ( 2 vs. 8 sec) and motion (grouped vs. separated). There were main effects of cue $[F(1,122)=205.225, p<$ $\left..001, \eta_{\mathrm{p}}^{2}=.627\right]$, task $\left[F(1,122)=9.71, p<.003, \eta_{\mathrm{p}}^{2}=\right.$ $.074]$, and motion $\left[F(1,122)=7.75, p<.007, \eta_{\mathrm{p}}^{2}=.06\right]$, as well as a cue $\times$ task interaction $[F(1,122)=10.653$, $\left.p<.002, \eta_{\mathrm{p}}^{2}=.08\right]$. None of the other effects was significant, with the cue $\times$ motion interaction having the highest $F$ of $3.17\left(p>.07, \eta_{\mathrm{p}}^{2}=.025\right)$. The unexpected main effect of motion was due to higher ratings in the grouped-motion condition $(M=7.45)$ than in the separated-motion condition $(M=7.18)$. 
Separated
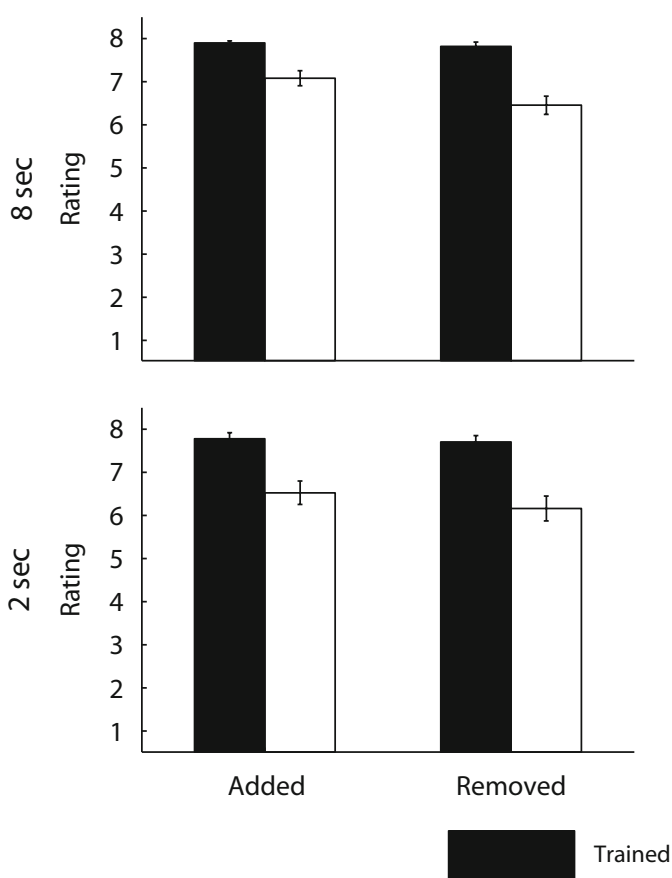

Grouped
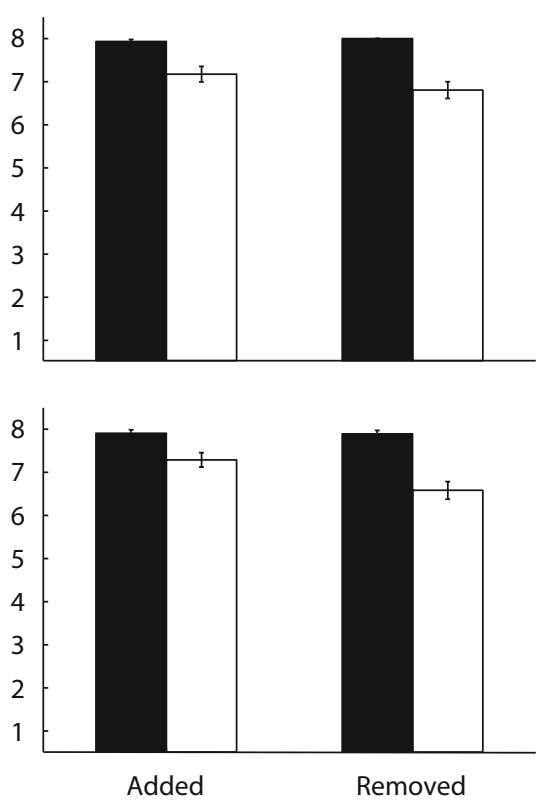

Test

Figure 1. Mean ratings of the reinforced trained stimuli (AB in the added-cue task and EFG in the removed-cue task) and the corresponding test stimuli (ABX or EF/EG/FG, respectively) in the test stage of Experiment 1 for the four conditions.

The present experiment revealed asymmetrical decrements: Removing an already trained component from a three-dot compound resulted in larger decrements than adding a new one to a two-dot compound. In our previous experiments, we had observed reliable symmetrical decrements in human predictive learning, using the same kind of stimulus material and a stimulus duration of $2 \mathrm{sec}$. We had hypothesized that the symmetrical decrements observed by Thorwart and Lachnit (2009) were due to time pressure during stimulus processing. However, stimulus duration in the present experiment had no effect on the decrements' symmetry. Decrements were asymmetrical with a stimulus duration of $2 \mathrm{sec}$, as well as with one of $8 \mathrm{sec}$. Therefore, time pressure is not sufficient to induce symmetrical decrements.

Consistent with Experiment 1 of Thorwart and Lachnit (2009), the kind of motion did not influence the decrements' symmetry (or asymmetry). Since decrements in groups with separated motion were asymmetrical in the present experiment, there was no floor effect here and we have to conclude that the motion, grouped or separated, is also insufficient for symmetrical decrements.

The simplest explanation for the differing results so far is that a causal cover story is necessary and sufficient for asymmetrical decrements. In Thorwart and Lachnit (2009), a power plant scenario, without any information about the causal nature of the link between the cues and the outcome, was used. In the present study, the instruction highlighted the causal relationship between the bacteria and the disease as outcome. This conclusion is supported by the results of previous causal learning stud- ies of Wheeler et al. (2006) and Glautier (2004), which had observed asymmetrical decrements. Furthermore, this would be in line with the suggestion of Pineño et al. (2005) that there is greater susceptibility to cue competition in causal than in predictive attribution. Note that this could yield a purely elemental explanation of the symmetry observed in Thorwart and Lachnit. In elemental models, the decrement in the removed-cue task is due to overshadowing - that is, cue competition during the training stage - whereas the decrement in the addedcue task is based on changes in stimulus representation in the test stimuli. Following Pineño et al., changing the predictive cover story to a causal one should then result in an increased decrement for the removed-cue task but not for the added-cue task. In configural models, both decrements are due to a decreased activation of the previously trained configural unit. Changing the cover story should not have any differential effect on the two tasks. An influence of the cover story on the decrements then would imply that both symmetry and asymmetry could be due to stimulus processing following principles incorporated in elemental models. On the other hand, the differing susceptibility to cue competition is based only on the observation of Pineño et al. and, as they pointed out, cannot be explained by any contemporary-elemental or configural - model of associative learning in its current form. For example, Wagner's (2008) REM has a parameter accounting for perceptual interactions $(r=$ proportion of context-dependent elements) that influence the amount of overshadowing but not the asymmetry of the decrements. Therefore, an influence of the cover story 
on the asymmetry would pose a major challenge to all current associative learning models. In Experiment 2, we looked for further empirical evidence.

\section{EXPERIMENT 2}

The following experiment investigated whether the causal cover story influenced the symmetry of the generalization decrements in Experiment 1. One group received the instructions used in Experiment 1. Participants were told that they had to learn which bacteria in blood samples are causal for a new disease. The instruction of Group Predictive stated that participants will learn which components of a blood sample are indicators for a new disease and allow diagnosis of it. Furthermore, the rating question of Group Causal asked "Are the bacteria in this blood sample causing the disease?" whereas Group Predictive was asked "Are the components in this blood sample indicating a disease?"

Because Experiment 1 replicated Thorwart and Lachnit (2009) in finding no influence of perceptual grouping by motion of and revealed no effect of stimulus duration, the dots in the following experiment were displayed on fixed positions in the middle of the screen for $2.5 \mathrm{sec}$, in a manner similar to the procedure of Experiment 3 of Thorwart and Lachnit.

\section{Method}

Only differences from the previous experiment are reported.

Participants. Forty-three of 63 participants were included in the analyses, having successfully solved the discrimination during training. They were from 19 to 34 years of age, with a mean age of 21.3. Thirteen men and 30 women took part; 21 participants were tested with predictive instruction and 22 with the causal instruction.

Apparatus and Stimuli. The experiment took place in labs in the Department of Psychology or in a room of the university's student services house. In the student services house, an IBM Thinkpad placed in a paperboard cabin was used. All monitors were 15 in. and running in a 1,024 (width) $\times 768$ (height) pixel resolution with a $60-\mathrm{Hz}$ frame rate. The participants wore sound-attenuating earmuffs. The 16-pixel dots were presented on a horizontal line in the middle of the screen for 150 frames, resulting in a 2.5 -sec stimulus presentation. The colored dots were randomly assigned to a position in each trial.
Procedure. The following instruction was presented in Group Predictive, with changes to the causal instruction in boldface:

Dear participant! Thank you for your readiness to participate in this experiment.

In the following experiment, you will learn which components in the blood are indicators for a new illness and thus allow its diagnosis. Please imagine that you are working in a medical laboratory. Recently there were several cases of this new illness. In order to diagnose this illness, you use a new procedure for investigating blood samples.

At any one time, you will see the sample of one test person on the screen. Afterward please indicate whether the blood components in this blood sample indicate a disease, and therefore whether the test person has gotten sick or not. Perhaps you will not be perfectly sure with your decision; therefore, an 8-point answer scale is available, ranging from " $1=$ definitely not ill" to " $8=$ definitely ill." After you have delivered your rating, you will receive feedback whether the disease occurred or not. At the beginning you will have to guess but after a while you will learn what are indicators for a disease. It doesn't matter how fast you answer; therefore, take the time you need. If you have any question, please ask now. Otherwise, please start the experiment by clicking on the "next" button.

The rating question asked "Do the components in this blood sample indicate a disease?"

\section{Results and Discussion}

Figure 2 displays the mean rating for the relevant stimuli in the test stage. The left panel shows the results of the group with a causal cover story. The group with a predictive cover story is shown on the right side. Again, within each panel, the black bars represent the mean rating of the previously trained stimulus $\mathrm{AB}$ for the added-cue task on the left side and EFG for the removed-cue task on the right side), whereas the white bars represent the averaged rating of the corresponding test stimulus ( $\mathrm{ABX}$ and $\mathrm{EF} / \mathrm{EG} / \mathrm{FG}$, respectively).

In both groups, the ratings of test stimuli were lower than those of the reinforced training stimuli. As in Experiment 1 , the decrement seemed to be larger for the removed-cue than for the added-cue task. This was con-
Causal



Predictive

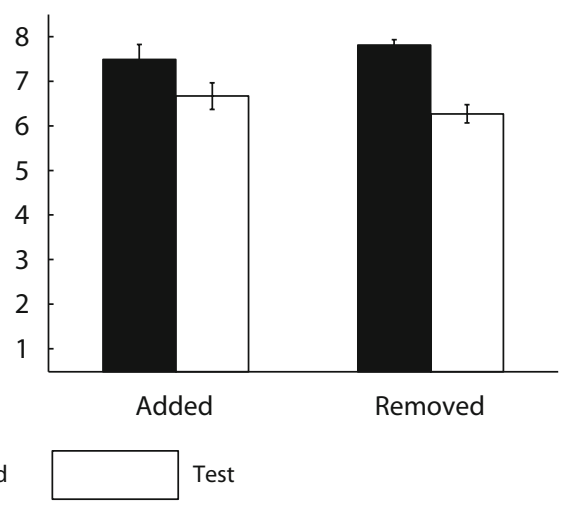

Figure 2. Mean ratings of the reinforced trained stimuli (AB in the added-cue task and EFG in the removed-cue task) and the corresponding test stimuli (ABX or EF/EG/FG, respectively) in the test stage of Experiment 2 for the two instructions. 
firmed by a $2 \times 2 \times 3 \times 2$ ANOVA including the withinsubjects factors cue (trained vs. test), task (added vs. removed), and block (1-3) and the between-subjects factor cover story (causal vs. predictive). There were main effects of cue $\left[F(1,41)=61.00, p<.001, \eta_{\mathrm{p}}^{2}=.598\right]$, and a cue $\times$ task interaction $\left[F(1,41)=4.09, p<.05, \eta_{\mathrm{p}}^{2}=\right.$ .091]. None of the other effects was significant, with the main effect of instruction having the highest $F$ of 2.973 $\left(p>.09, \eta_{\mathrm{p}}^{2}=.068\right)$.

Again, we found asymmetrical generalization decrements, as in Experiment 1. In contrast to Pineño et al. (2005), we found that the cover story had no differential effect on the amount of overshadowing in the removed-cue task, and therefore on the asymmetry of the decrements. The divergence between Experiment 1 and the previous experiments of Thorwart and Lachnit (2009) cannot be traced back to the introduction of a causal cover story in the former. In other words, symmetrical decrements cannot be attributed solely to the fact that a predictive cover story reduces the susceptibility to cue competition effects, because we now observed both symmetry and asymmetry within the context of a predictive cover story.

\section{GENERAL DISCUSSION}

In two experiments, we observed asymmetrical generalization decrements. This is in contrast to our previous finding (Thorwart \& Lachnit, 2009) but in line with other studies (e.g., Wheeler et al., 2006). This indicates that stimulus processing and representation differed between the experiments. As described above, asymmetrical decrements are in line with elemental models (Harris, 2006; Wagner, 2008), whereas the previously observed symmetry is captured only by models based on configural principles (Pearce, 1994). The experiments thereby provide further empirical support for the assumption that the representation of the cues in associative learning is flexible and, instead of contrasting configural and elemental accounts, research needs to shift its focus to the variables controlling the nature of representation and processing.

In Thorwart and Lachnit (2009), we discussed several potential reasons that might have caused the symmetrical decrements observed there. However, even if we observe the discrepancy between experiments, neither different degrees of time pressure during stimulus processing nor different degrees of perceptual grouping by motion proved effective in producing both symmetrical and asymmetrical decrements within the present Experiment 1. The same is true for the predictive versus causal nature of the relationship between the stimuli in Experiment 2. Although we acknowledge that we cannot provide an independent validation of our manipulations, it seems appropriate to conclude that the previously observed effects of these manipulations in other learning tasks are not as reliable and general as one might have thought. Lachnit et al. (2010) observed, for example, that introducing time pressure in a causal learning experiment reversed the rank order of the acquisition of two discriminations, compared with an experiment in which the participants paced themselves. Karazinov and Boakes (2007) investigated the relation- ship of time pressure in the switch between second-order conditioning and inhibition learning in feature-negative tasks and observed second-order conditioning under a 3-sec paced condition, even in an intermixed featurenegative design. Glautier (2002) observed no blocking when stimuli were presented on the same location, compared with spatially separated stimuli, whereas Livesey and Boakes (2004) observed blocking effects with a nonadditive pretraining when the spatial angle of two stimuli was large. They further observed that presenting the stimuli in a way designed to favor configural processing (chemical substances in one container) resulted in the absence of blocking despite additivity training. Concerning the nature of the cover story, Pineño et al. (2005)'s experiments have already been mentioned. These examples of successful manipulation indicate that more controlled research is needed, to investigate not only the appropriate operationalization of these critical factors, but also the circumstances controlling the factors' relevance in human associative learning.

For the results presented in the present article, the million-dollar question, then, is to explain the remaining discrepancy between the experiments. The divergence, in particular, is astonishing, when the present Experiment 2 is compared with Thorwart and Lachnit's (2009) Experiment 3 . The same kind of stimuli and training schedule were used in the present Experiment 2 as in the fixed group of Thorwart and Lachnit's Experiment 3. The same is true for the present Experiment 1 and Experiment 1 of Thorwart and Lachnit, both of which investigated perceptual grouping by motion and used the same moving dots. Of course, there are still differences in the procedures (e.g., the scenario chosen, the speed of the moving dots, the test trials, the number of colors presented throughout the training), but there are neither empirical nor theoretical grounds for thinking that any of these is likely to be a critical factor in controlling the nature of stimulus representation and processing. In the end, we have to state that, at the moment, we do not know what the critical variables may be that provoke reliable symmetrical decrements in Thorwart and Lachnit, but provoke reliable asymmetrical decrements in the present experiments.

\section{AUTHOR NOTE}

This research is part of the doctoral thesis of A.T. She was supported by the DFG (Deutsche Forschungsgemeinschaft) graduate program NeuroAct (DFG 885), and H.L. was supported by Grant La 564/19-1. We thank an anonymous reviewer for valuable and constructive comments on an earlier version. Correspondence concerning this article should be addressed to A. Thorwart, School of Psychology, Brennan MacCallum Building (A18), University of Sydney, NSW 2006, Australia (e-mail: anna.thorwart@sydney.edu.au)

\section{REFERENCES}

Brainard, D. H. (1997). The Psychophysics Toolbox. Spatial Vision, 10, 433-436.

Brandon, S. E., Vogel, E. H., \& Wagner, A. R. (2000). A componential view of configural cues in generalization and discrimination in Pavlovian conditioning. Behavioural Brain Research, 110, 67-72.

Giurfa, M., Schubert, M., Reisenman, C., Gerber, B., \& LachNIT, H. (2003). The effect of cumulative experience on the use of elemental and configural visual discrimination strategies in honey- 
bees. Behavioural Brain Research, 145, 161-169. doi:10.1016/S0166 $-4328(03) 00104-9$

Glautier, S. (2002). Spatial separation of target and competitor cues enhances blocking of human causality judgements. Quarterly Journal of Experimental Psychology, 55B, 121-135. doi:10.1080/ 02724990143000207

Glautier, S. (2004). Asymmetry of generalization decrement in causal learning. Quarterly Journal of Experimental Psychology, 57B, 315329. doi: $10.1080 / 02724990344000169$

Glautier, S., Redhead, E., Thorwart, A., \& Lachnit, H. (2010). Reduced summation with common features in causal judgments. Experimental Psychology, 57, 252-259.

González, F., Quinn, J. J., \& Fanselow, M. S. (2003). Differential effects of adding and removing components of a context on the generalization of conditional freezing. Journal of Experimental Psychology: Animal Behavior Processes, 29, 78-83. doi:10.1037/0097 $-7403.29 .1 .78$

HARRIS, J. A. (2006). Elemental representations of stimuli in associative learning. Psychological Review, 113, 584-605. doi:10.1037/0033 $-295 X .113 .3 .584$

Harris, J. A., Livesey, E. J., Gharaei, S., \& Westbrook, R. F. (2008). Negative patterning is easier than a biconditional discrimination. Journal of Experimental Psychology: Animal Behavior Processes, 34, 494-500. doi:10.1037/0097-7403.34.4.494

HuYNH, H., \& FeldT, L. S. (1976). Estimations of the Box correction for degrees of freedom from sample data in randomized block and split-plot designs. Journal of Educational Statistics, 1, 69-82.

Karazinov, D. M., \& Boakes, R. A. (2007). Second-order conditioning in human predictive judgements when there is little time to think. Quarterly Journal of Experimental Psychology, 60, 448-460. doi:10.1080/17470210601002488

Kinder, A., \& LaCHNit, H. (2003). Similarity and discrimination in human Pavlovian conditioning. Psychophysiology, 40, 226-234.

LACHNIT, H. (1988). Convergent validation of information processing constructs with Pavlovian methodology. Journal of Experimental Psychology: Human Perception \& Performance, 14, 143-152.

Lachnit, H., Schultheis, H., KöNIG, S., ÜNGÖr, M., \& Melchers, K. G. (2008). Comparing elemental and configural associative theories in human causal learning: A case for attention. Journal of Experimental Psychology: Animal Behavior Processes, 34, 303-313. doi:10.1037/0097-7403.34.2.303

Lachnit, H., Thorwart, A., Schultheis, H., Üngör, M., Lotz, A., \& KöNIG, S. (2010). Indicators of early and late processing reveal the importance of within-trial-time for theories of associative learning. Manuscript in preparation.

Livesey, E. J., \& Boakes, R. A. (2004). Outcome additivity, elemental processing and blocking in human causality judgements. Quarterly Journal of Experimental Psychology, 57B, 361-379. doi:10.1080/02724990444000005

Mackintosh, N. J. (1975). A theory of attention: Variations in the associability of stimuli with reinforcement. Psychological Review, 82, 276-298.

Melchers, K. G., Shanks, D. R., \& Lachnit, H. (2008). Stimulus coding in human associative learning: Flexible representations of parts and wholes. Behavioral Processes, 77, 413-427. doi:10.1016/ j.beproc.2007.09.013

Myers, K. M., Vogel, E. H., Shin, J., \& Wagner, A. R. (2001). A comparison of the Rescorla-Wagner and Pearce models in a negative patterning and a summation problem. Animal Learning \& Behavior, 29, 36-45.

Palmer, S. E. (1999). Vision science: Photons to phenomenology. Cambridge, MA: MIT Press

Pearce, J. M. (1987). A model for stimulus generalization in Pavlovian conditioning. Psychological Review, 94, 61-73.
Pearce, J. M. (1994). Similarity and discrimination: A selective review and a connectionist model. Psychological Review, 101, 587-607.

Pearce, J. M., Aydin, A., \& Redhead, E. S. (1997). Configural analysis of summation in autoshaping. Journal of Experimental Psychology: Animal Behavior Processes, 23, 84-94.

Pearce, J. M., \& Bouton, M. E. (2001). Theories of associative learning in animals. Annual Review of Psychology, 52, 111-139.

Pearce, J. M., \& Hall, G. (1980). A model for Pavlovian conditioning: Variations in the effectiveness of conditioned but not of unconditioned stimuli. Psychological Review, 87, 532-552.

Pineño, O., Denniston, J. C., Beckers, T., Matute, H., \& Miller, R. R. (2005). Contrasting predictive and causal values of predictors and of causes. Learning \& Behavior, 33, 184-196.

Redhead, E. S., \& Pearce, J. M. (1995). Similarity and discrimination learning. Quarterly Journal of Experimental Psychology, 48B, 46-66.

Rescorla, R. A. (1999). Associative changes in elements and compounds when the other is reinforced. Journal of Experimental Psychology: Animal Behavior Processes, 25, 247-255.

Rescorla, R. A., \& Wagner, A. R. (1972). A theory of Pavlovian conditioning: Variations in the effectiveness of reinforcement and nonreinforcement. In A. H. Black \& W. F. Prokasy (Eds.), Classical conditioning II: Current theory and research (pp. 64-99). New York: Appleton-Century-Crofts.

Schultheis, H., Thorwart, A., \& Lachnit, H. (2008a). HMS: A MATLAB simulator of the Harris model of associative learning. Behavior Research Methods, 40, 442-449. doi:10.3758/BRM.40.2.442

Schultheis, H., Thorwart, A., \& Lachnit, H. (2008b). Rapid-REM: A MATLAB simulator of the replaced-elements model. Behavior Research Methods, 40, 435-441. doi:10.3758/BRM.40.2.435

Thorwart, A., Glautier, S., \& Lachnit, H. (2010). Convergent results in eyeblink conditioning and contingency learning in humans: Addition of a common cue does not affect feature-negative discriminations. Biological Psychology, 85, 207-212. doi:10.1016/ j.biopsycho.2010.07.002

THORWART, A., \& LACHNit, H. (2009). Symmetrical generalization decrements: Configural stimulus processing in human contingency learning. Learning \& Behavior, 37, 107-115. doi:10.3758/LB.37.1.107

Thorwart, A., Schultheis, H., König, S., \& Lachnit, H. (2009). ALTSim: A MATLAB simulator for current associative learning theories. Behavior Research Methods, 41, 29-34. doi:10.3758/BRM.41.1.29

WAGNER, A. R. (2008). Evolution of an elemental theory of Pavlovian conditioning. Learning \& Behavior, 36, 253-265. doi:10.3758/LB.36 .3 .253

Wasserman, E. A., \& Miller, R. R. (1997). What's elementary about associative learning? Annual Review of Psychology, 48, 573-607. doi:10.1146/annurev.psych.48.1.573

Wheeler, D. S., Amundson, J. C., \& Miller, R. R. (2006). Generalization decrement in human contingency learning. Quarterly Journal of Experimental Psychology, 59, 1212-1223. doi:10.1080/ 17470210600576342

\section{NOTE}

1. However, Rescorla (1999) reported symmetrical generalization decrements in several experiments that compared forward and backward blocking. There, the first trials of Stage 2 measure nothing other than decrements after adding or removing a component. Furthermore, Pearce (1987) cited Young (unpublished dissertation), who observed no differences in extinction after adding or removing a component from the trained stimulus compound in the extinction phase.

(Manuscript received February 8, 2010; revision accepted for publication March 7, 2010.) 